\title{
Role of tissue transglutaminase and effect of cantharidinate in human colorectal cancer
}

\author{
LAN-LAN YANG ${ }^{1}$, CHONG-YANG LIANG $^{2}$, TIAN-CHENG LU ${ }^{3}, \mathrm{CHEN}$ YANG ZHI $^{4}$, BIN LIU $^{5}$, \\ JIAN-HUA ZHOU ${ }^{3}, \mathrm{XU}_{-M I N G ~ \text { LIU }^{4}, \text { HAI-CHENG GAO }}^{2}$ and WEI HUANG ${ }^{6}$ \\ ${ }^{1}$ Department of Gastroenterology and Hepatology, The Second Hospital of Jilin University, Changchun, Jilin 130041; \\ ${ }^{2}$ Department of Clinical Pharmacy and Pharmaceutical Management, School of Pharmaceutical Sciences, \\ Jilin University, Changchun, Jilin 130021; ${ }^{3}$ School of Life Sciences, Jilin Agricultural Univesity, Changchun, Jilin 130118; \\ ${ }^{4}$ Changchun University of Traditional Chinese Medicine, Changchun, Jilin 130118; ${ }^{5}$ Department of General Surgery, \\ Jilin University Second Hospital, Changchun, Jilin 130041; ${ }^{6}$ Department of Radiotherapy, \\ Tumor Center, The First Hospital of Jilin University, Changchun, Jilin 130021, P.R. China
}

Received March 23, 2013; Accepted September 17, 2013

DOI: $10.3892 / \mathrm{mmr} .2013 .1706$

\begin{abstract}
The role of tissue transglutaminase (tTG) in cancer development remains an important field of study. The aim of the current study was to understand the involvement of tTG in cancer and the inhibitory effect of cantharidinate on the expression of tTG in human colorectal cancer (CRC) using immunohistochemical and PCR analysis. The results showed that the expression of tTG increased in human CRC and cantharidinate inhibited the expression of tTG. These results suggested that tTG is significant in human CRC and that tTG may be an important target for tumor chemoprevention and treatment. Cantharidinate may be considered as a novel co-therapy for controlling tTG expression in human CRC.
\end{abstract}

\section{Introduction}

Human colorectal cancer (CRC) is a type of cancer that arises from uncontrolled cell growth in the colon or rectum, sections of the large intestine or in the appendix (1). Genetic analysis shows that colon and rectal tumors are essentially the same type of cancer (2). CRC is the third most commonly diagnosed type of cancer in males and the second in females, and in 2008 there was an estimated 1.2 million novel CRC cases and 608,700 mortalities $(3,4)$. Symptoms of CRC typically include rectal bleeding and anemia, which may be associated with weight loss and changes in bowel habits (5). The majority

Correspondence to: Dr Hai-Cheng Gao or Dr Chong-Yang Liang, Department of Clinical Pharmacy and Pharmaceutical Management, School of Pharmaceutical Sciences, Jilin University, 1163 Xinmin Street, Changchun, Jilin 130021, P.R. China

E-mail: gaohc2012@sina.com; gaohc@jlu.edu.cn

Key words: colorectal cancer, tissue transglutaminase, molecular target, cantharidinate of CRC cases are a result of lifestyle and increasing age, with a minority of cases associated with underlying genetic disorders $(6,7)$. Cancers confined within the wall of the colon are often curable with surgery, while cancers that have metastasized around the body are usually incurable. At present, studies primarily focus on tumor chemotherapy (8); however, the pathogenesis of CRC remains unclear. Current management focuses on extending patient lifespan via chemotherapy and improving quality of life (9).

Tissue transglutaminase (tTG) is a matricellular protein, which is expressed in multiple tissue and cell types (10). It is unclear whether tTG is important in CRC and if so, whether tTG may be inhibited by certain of drugs. Fluorouracil is a standard chemotherapeutic agent used in the treatment of CRC (11). Treatment options for CRC also include additional chemotherapeutic agents and targeted therapies, including cetuximab, panitumumab and bevacizumab (12). Correct use of these therapies may result in a positive impact on patient prognosis (13).

Previously, data concerning cancer treatment using traditional Chinese medicine has affected a number of cancer treatments (14). It remains unclear whether certain traditional Chinese medicines may be used to inhibit tTG expression and improve the symptoms of CRC. Cantharidin (also termed cantharidinate) has been used in traditional Chinese medicine $(15,16)$. Cantharidinate induces cell cycle arrest and triggers apoptosis in various types of tumor cells, including hepatoma, myeloma, oral buccal carcinoma, leukemia, gastric cancer, human bladder carcinoma TSGH-8301, colorectal cancer colo 205, A549 human lung cancer and intestinal epithelial cells (17-20). Therefore, the present study investigated the involvement of tTG in human CRC and demonstrated that cantharidinate may be used to inhibit the expression of tTG.

\section{Materials and methods}

Patients and tissue specimens. Twenty human CRC tissue specimens were obtained from 12 males and 8 females, 
(average age, 68.25 years; range, 21-87 years) by surgical resection between May 2011 and June 2012 in Jilin University Second Hospital (Jilin, China). Written informed patient consent was obtained and approval was acquired from the Jilin University Second Hospital Ethics Committee (no. 2012-43). Tissue microarrays (TMAs) were constructed. Histological tumor grade of colon and sigmoid colon tissue were included in the analysis. Expression of tTG was associated with patient demographics, adjuvant treatment regimens and histological parameters.

Histopathologic examination. Specimens and cells were examined using light microscopy (Eclipse TE-2000-U equipped with an attached SXM1200F digital camera; Nikon, Tokyo, Japan) following haematoxylin and eosin (HE) staining.

Immunohistochemical staining. Paraffin-embedded slices (4- $\mu \mathrm{m}$ thick) were probed with anti-human $\mathrm{tTG}$ monoclonal antibody (Sigma-Aldrich, St. Louis, MO, USA; dilution, 1:300) at $4^{\circ} \mathrm{C}$ overnight. Sections were immersed in $0.3 \% \mathrm{H}_{2} \mathrm{O}_{2}$ in absolute methanol for 15 min to block endogenous peroxidase. Color was developed using chromagen 3,3'-diaminobenzidine (DAB substrate kit and Immunohistochemistry kit; Biosynthesis Biotechnology, Beijing, China). Slices were counterstained with hematoxylin, mounted on glass coverslips and sealed with neutral resin.

UCT-116 cell culture and treatment. UCT-116 human CRC cell lines were donated from Jilin University Institute of Regenerative Medicine. UCT-116 cells were routinely cultured in Dulbecco's modified Eagle's media (DMEM; Gibco-BRL, Life Technologies, Carlsbad, CA, USA) supplemented with $20 \%$ fetal bovine serum (FBS; Gibco-BRL) and $50 \mathrm{U} / \mathrm{ml}$ antibiotics under the conditions of $5 \% \mathrm{CO}_{2}$ at $37^{\circ} \mathrm{C}$. Following trypsinization, cells were incubated in DMEM with $0.5 \%$ FBS for $24 \mathrm{~h}$. Cells were treated with $2.5 \mu \mathrm{mol} / \mathrm{l}$ cantharidinate, provided by Associate Professor Yang of the Second Hospital of Jilin University and $2.5 \mu \mathrm{mol} / 1$ fluorouracil (Shanghai Hanhong Group, Shanghai, China), with untreated cells used as a control, for $48 \mathrm{~h}$.

Quantitative PCR (qPCR) analysis of UCT-116 cells. Total RNA was extracted using TRIzol reagent (Invitrogen Life Technologies, Carlsbad, CA, USA) according to the manufacturer's instructions. First strand cDNA was synthesized using PrimeScript ${ }^{\mathrm{TM}} \mathrm{RT}$ enzyme mix I, oligo dT primers and random hexamers (Takara Bio, Inc., Shiga, Japan). qPCR analysis was performed using first strand cDNA, forward and reverse primers and the SYBR premix Ex Taq ${ }^{\mathrm{TM}}$ Green II kit (Takara). Primers were synthesized by Sangon Biotech Company (Shanghai, China) and the sequences were as follows: Forward: 5'-GGCACAGTCAAGGCTGAGAATG-3' and reverse: 5'-ATGGTGGTGAAGACGCCAGTA-3' for glyceraldehyde 3-phosphate dehydrogenase (GAPDH) and forward: 5'-GACAAGCGCATCACACAGACA-3' and reverse: 5'-TCTTTCGTTAGAGCCAAGGCC-3' for tTG. Reaction and signal detection were measured in triplicate independently by an iCyder iQ real-time PCR Detection System (Bio-Rad, Hercules, CA, USA). mRNA levels were calculated as the relative expression ratio compared with GAPDH.
Table I. Clinicopathological features of colon cancer patient cohort $(n=20)$.

Male $(\mathrm{n}=12) \quad$ Female $(\mathrm{n}=8)$

\begin{tabular}{lcc}
\hline Age (years) & & \\
Mean & 63.5 & 73.375 \\
Range & $21-80$ & $63-87$ \\
Minimum & $21(12.5 \%)$ & $63(25 \%)$ \\
Maximum & $80(12.5 \%)$ & $87(37.5 \%)$ \\
Grade of differentiation & & \\
IIB & $1(8.3 \%)$ & 0 \\
IIC & $1(8.3 \%)$ & 0 \\
IIIB & $1(8.3 \%)$ & $2(25 \%)$ \\
IIIA & 0 & $1(12.5 \%)$ \\
A moderately differentiated & $9(75 \%)$. & $5(40 \%)$ \\
adenocarcinoma & & \\
\hline
\end{tabular}

Statistical analysis. Statistical analysis of data was performed using SPSS Version 11 for Windows (SPSS, Inc., Chicago, IL, USA). All data are presented as the mean \pm SEM. Statistical comparisons were determined by Student's t-test and $\mathrm{P}<0.05$ was considered to indicate a statistically significant difference.

\section{Results}

Clinicopathological features and patient outcome. The present study was performed on a TMA, constructed from surgically resected samples of patients with varying grades of differentiation of CRC. The demographics of the patients are shown in Table I as well as the clinicopathological features of the colon and the grade of differentiation. The incidence of CRC of grades IIB, IIC, IIIB and IIIA in males was $8.3,8.3,8.3$ and $0 \%$, respectively. The incidence of CRC of grades IIB, IIC, IIIB and IIIA in females was 0, 0, 25 and $12.5 \%$, respectively. The incidence of colon and sigmoid colon cancer was 75 and $25 \%$ in males and 40 and $60 \%$ in females, respectively.

$H \& E$ of human CRC tissues. Following standard H\&E staining, normal colon tissue and the CRC TMAs were observed to have representative histological structures by microscopy (Fig. 1). The immunohistochemical staining of microarray samples was representative (Fig. 2A and B).

Immunohistochemical staining of tTG in human CRC tissue. Immunohistochemical staining was used to determine the expression of tTG protein in human CRC tissue. The results showed that tTG was expressed in the membrane and cytoplasm of the normal tissue of patients with CRC (Fig. 2A). The expression of tTG markedly increased in CRC tissue. tTG was primarily expressed in the tumor and interstitial regions (Fig. 2B). The expression of tTG exhibited a significant difference between $\mathrm{CRC}$ and normal tissue $(\mathrm{P}<0.01$; Fig 2C). 


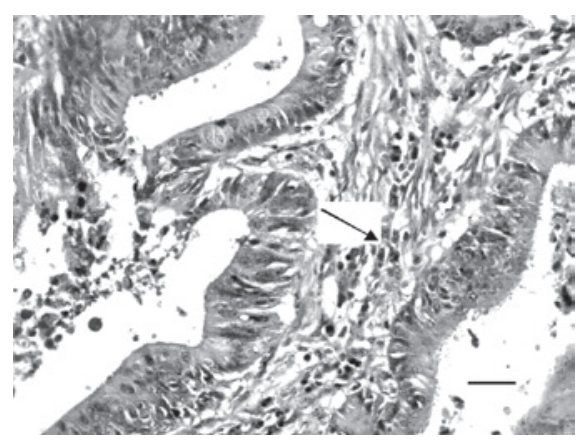

Figure 1. Pathological examination of a human CRC specimen from a 60 -year-old male by hematoxylin and eosin staining. Histological analysis showed highly differentiated CRC where atypic glands were arranged in a dense formation and cancer cells exhibited large and deeply-stained disordered nuclei. Scale bar, $5 \mu \mathrm{m}$. CRC, colorectal cancer.

A

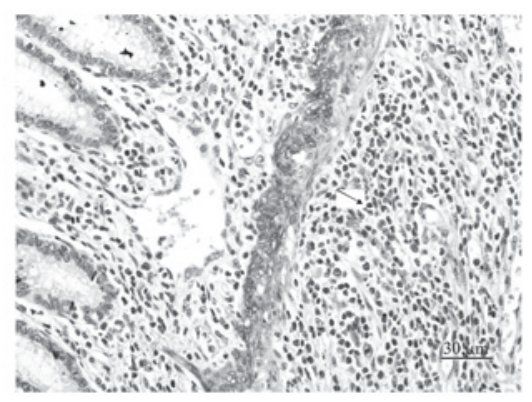

B

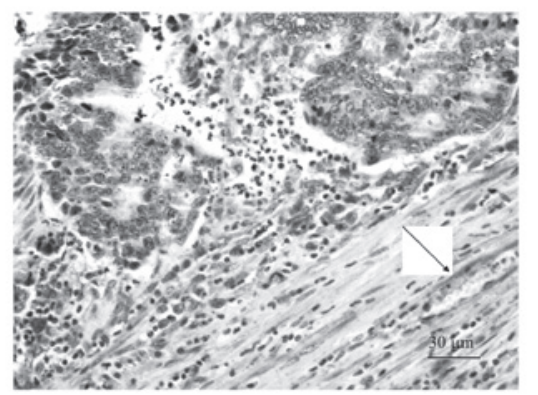

C

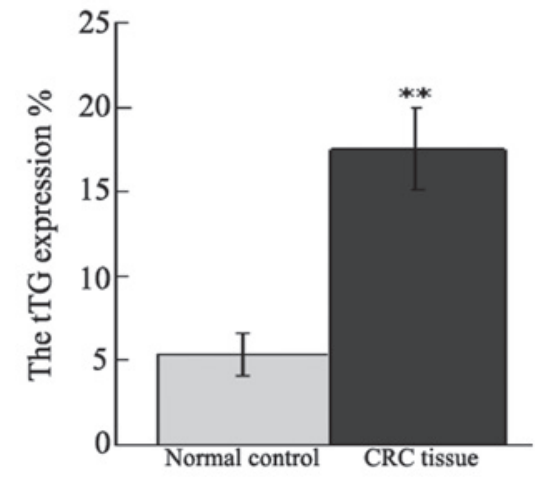

Figure 2. Immunohistochemical staining of (A) peripheral normal control tissue and (B) colorectal tissue with increased tissue tTG expression. Positive tTG expression was shown by brown/yellow immunohistochemical staining. (C) Semiquantified levels of tTG protein in colorectal cancer (CRC) tissue were increased significantly compared with the normal control. ${ }^{* *} \mathrm{P}<0.01$, vs. control as determined by Student's t-test. tTG, transglutaminase.

H\&E staining of UCT-116 cells. Pathological changes of UCT-116 human CRC cells in various groups are shown in Fig. 3A-C. The quantity of UCT-116 cells decreased $48 \mathrm{~h}$
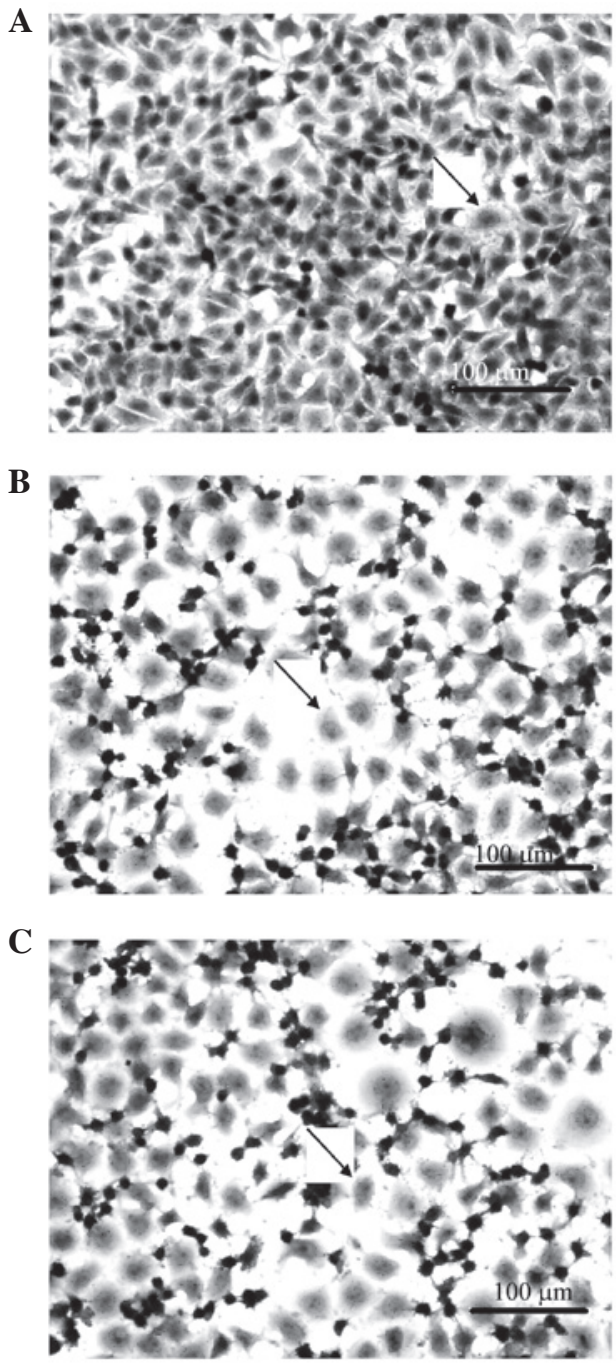

Figure 3. Hematoxylin and eosin staining in (A) control, (B) fluorouracil-treated and (C) cantharidinate-treated UCT-116 human colorectal cancer cells. Fluorouracil and cantharidinate markedly reduced the number of cancer cells.

following the application of fluorouracil and cantharidinate (Fig. 3B and C). This result suggested that cantharidinate may exert an accessorial effect and may reduce the chemotherapeutic time, subsequently decreasing the side-effects of the treatment of human CRC.

Immunohistochemical staining detects tTG in UCT-116 cells. Fig. 4 shows tTG immunohistochemical staining in UCT-116 cells. Immunohistochemical staining detected a high level of tTG in the untreated UCT-116 cell control (Fig. 4A and D). Cantharidinate and fluorouracil treatment decreased the level of tTG in UCT-116 cells significantly ( $\mathrm{P}<0.05$; Fig. 4B-D). This result suggested that cantharidinate may inhibit the expression of tTG and block tumor growth.

PCR analysis results. To determine whether the tTG mRNA level in UCT-116 cells altered following the application of cantharidinate, qPCR analysis was performed. In Fig. 5, the expression of tTG mRNA in the cantharidinate group decreased to 0.69 -fold that of the untreated cancer cell control $(\mathrm{P}<0.05)$. The level of tTG in the cantharidinate group was 


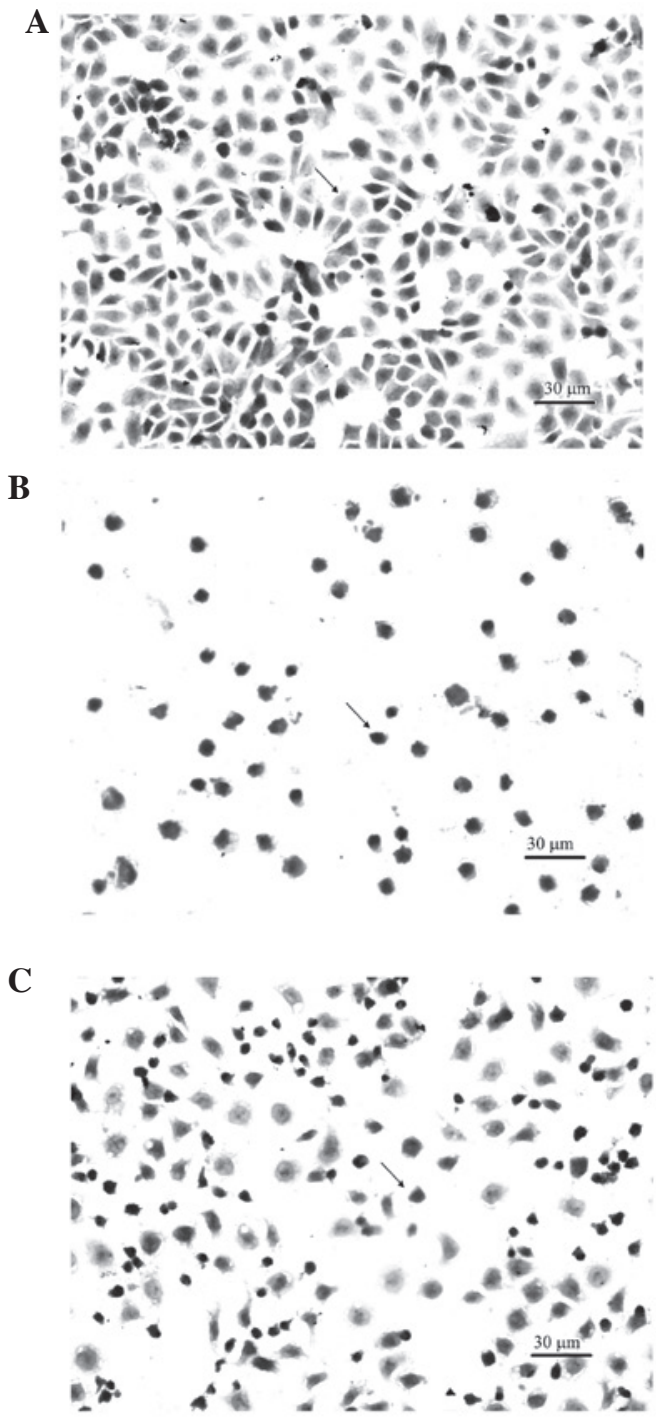

D

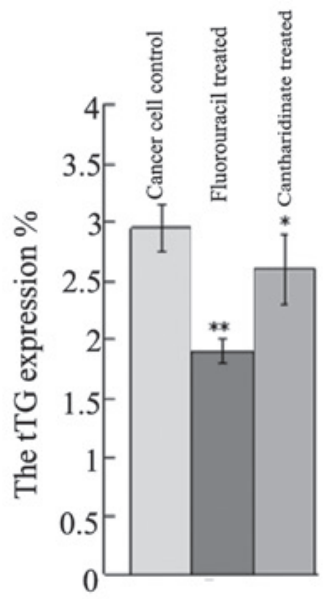

Figure 4. Immunohistochemical staining of tissue tTG in (A) control, (B) fluorouracil-treated and (C) cantharidinate-treated UCT-116 human colorectal cancer cells. Positive tTG expression was shown by brown/yellow immunohistochemical staining. (D) Semiquantified level of tTG protein showed fluorouracil-treated and cantharidinate-treated cells reduced the expression of tTG. ${ }^{* *} \mathrm{P}<0.01$ and ${ }^{*} \mathrm{P}<0.05$, vs. cancer cell control. tTG, transglutaminase.

approximately the same as that in the fluorouracil group. This result suggested that cantharidinate may kill tumor cells by inhibiting tTG mRNA expression.

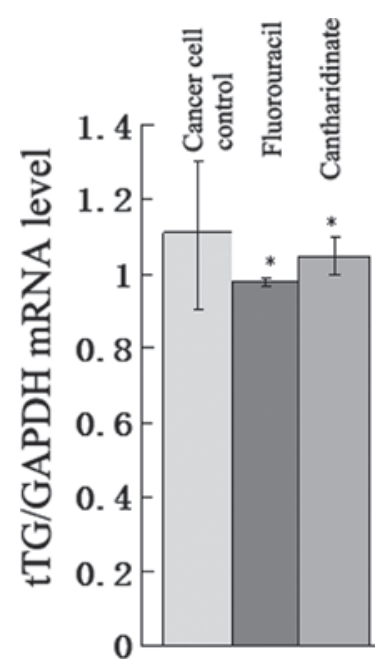

Figure 5. Quantitative PCR detects tTG mRNA levels in control, fluorouraciland cantharidinate treated UCT-116 human colorectal cancer cells. Treatment with fluorouracil and cantharidinate decreased the tTG mRNA level by 0.56- and 0.69-fold, respectively. ${ }^{*} \mathrm{P}<0.05$, vs. cancer cell control. tTG, transglutaminase; GAPDH, glyceraldehyde 3-phosphate dehydrogenase.

\section{Discussion}

tTGs are a family of structurally and functionally related proteins, which are widely distributed in all living organisms (10). There has been an increase in studies supporting the hypothesis that dysregulated expression and function of tTG contributes to pathological conditions, including cancer metastasis, tissue fibrosis, celiac disease, neurodegenerative disorders and diseases associated with the defective assembly of the stratum corneum of the skin (10). However, it remains unclear whether tTG is significant in CRC.

The present study demonstrated that levels of tTG were increased in the process of tumor occurrence and progression. The roles of tTG upregulation in cancers may involve the following. Agnihotri et al observed that tTG upregulation is associated with the activation of nuclear transcription factor- $\kappa \mathrm{B}$ $(\mathrm{NF}-\kappa \mathrm{B}), \mathrm{Akt}$, focal adhesion kinase and hypoxia-inducible factor, thus tTG may serve as a central mediator with a key role in the inflammation-induced progression of mammary epithelial cancer cells (21). Yakubov et al reported that extracellular tTG promotes the metastasis of ovarian cancer by noncanonical NF- $\kappa$ B activation (22). Furthermore, Caffarel et al demonstrated that tTG is able to promote the progression of cervical squamous cell carcinoma by enhancing the promalignant effects of oncostatin M receptor overexpression (23). Wang et al suggested that extracellular tTG has a crucial role in matrix-bound VEGF-mediated angiogenesis (24).

A desirable property of an anticancer drug is to induce the death of tumor cells with few side effects on normal cells $(12,13,15,16)$. The present study demonstrated that canthardinate induced an inhibitory effect on tTG, which is involved in human CRC. The effects of cantharidinate are similar to that of fluorouracil. Cantharidinate inhibits the proliferation of human CRC cells with an $\mathrm{IC}_{50}$ of $2.5 \mu \mathrm{M}$ and cantharidinate has marginal cytotoxicity in normal cells (data not shown). The present study demonstrated that cantharidinate reduced the expression of mRNA and protein levels of tTG in 
human CRC cells. To the best of our knowledge (15-20), the current study is the first to show that the expression of tTG mRNA and protein are downregulated by cantharidinate.

In conclusion, cantharidinate is significant in human CRC as it inhibits the expression of tTG. Therefore, cantharidinate may be considered to be a novel additional drug for controlling the expression of tTG in human CRC and the growth of human CRC.

\section{References}

1. Deng X, Cao Y, Liu Y, Li F, Sambandam K, Rajaraman S, Perkins AS, Fields AP, Hellmich MR, Townsend CM Jr, et al: Overexpression of Evi-1 oncoprotein represses TGF- $\beta$ signaling in colorectal cancer. Mol Carcinog 52: 255-264, 2013.

2. Potter JD: Colorectal cancer: molecules and populations. J Natl Cancer Inst 91: 916-932, 1999.

3. Deschoolmeester V, Baay M, Specenier P, Lardon F and Vermorken JB: A review of the most promising biomarkers in colorectal cancer: one step closer to targeted therapy. Oncologist 15: 699-731, 2010.

4. Kotzev I, Mirchev M, Manevska B, Ivanova I and Kaneva M: Risk and protective factors for development of colorectal polyps and cancer (Bulgarian experience). Hepatogastroenterology 55 381-387, 2008

5. Domínguez-Ayala M, Díez-Vallejo J and Comas-Fuentes A: Missed opportunities in early diagnosis of symptomatic colorectal cancer. Rev Esp Enferm Dig 104: 343-349, 2012.

6. Jass JR: Colorectal cancer: a multipathway disease. Crit Rev Oncog 12: 273-287, 2006.

7. Fearon ER: Molecular genetics of colorectal cancer. Annu Rev Pathol 6: 479-507, 2011.

8. Posner MR: Paradigm shift in the treatment of head and neck cancer: the role of neoadjuvant chemotherapy. Oncologist 10 (Suppl 3): 11-19, 2005

9. Simoglou C, Gymnopoulou E, Simoglou L, Gymnopoulou M, Nikolaou K and Gymnopoulos D: Surgery for colorectal cancer in the small town of Komotini. J Multidiscip Healthc 5: 273-276, 2012.

10. Hu Y, Zhang H, Xiong X, Cao Y, Han Y and Xi Z: Inhibitory effect of tissue transglutaminase (tTG) antisense oligodeoxynucleotides on tTG expression in cultured bovine trabecular meshwork cells. J Huazhong Univ Sci Technolog Med Sci 25: 729-731, 2005.

11. van Hazel GA, Pavlakis N, Goldstein D, Olver IN, Tapner MJ, Price D, Bower GD, Briggs GM, Rossleigh MA, Taylor DJ and George J: Treatment of fluorouracil-refractory patients with liver metastases from colorectal cancer by using yttrium-90 resin microspheres plus concomitant systemic irinotecan chemotherapy. J Clin Oncol 27: 4089-4095, 2009.
12. Gerber DE: Targeted therapies: a new generation of cancer treatments. Am Fam Physician 77: 311-319, 2008.

13. Dehmer GJ, Douglas JS Jr, Abizaid A, et al: SCAI/ACCF/HRS/ESC/SOLACI/APSIC statement on the use of live case demonstrations at cardiology meetings: assessments of the past and standards for the future. Heart Rhythm 7: 1522-1535, 2010.

14. Liu HG and Huang HX: Overview pharmacokinetic about traditional Chinese medicine in recent 10 years. Zhongguo Zhong Yao Za Zhi 32: 2346-2348, 2007 (In Chinese).

15. Honkanen RE: Cantharidin, another natural toxin that inhibits the activity of serine/threonine protein phosphatases types 1 and 2A. FEBS Lett 330: 283-286, 1993.

16. Deng LP, Dong J, Cai H and Wang W: Cantharidin as an antitumor agent: a retrospective review. Curr Med Chem 20: 159-166, 2013.

17. Huang YP, Ni CH, Lu CC, et al: Suppressions of migration and invasion by cantharidin in TSGH-8301 human bladder carcinoma cells through the inhibitions of matrix metalloproteinase-2/-9 signaling. Evid Based Complement Alternat Med 2013: 190281, 2013.

18. Zhan YP, Huang XE, Cao J, et al: Clinical study on safety and efficacy of Qinin ${ }^{\circledR}$ (cantharidin sodium) injection combined with chemotherapy in treating patients with gastric cancer. Asian Pac J Cancer Prev 13: 4773-4776, 2012.

19. Kim YM, Ku MJ, Son YJ, Yun JM, Kim SH and Lee SY: Anti-metastatic effect of cantharidin in A549 human lung cancer cells. Arch Pharm Res 36: 479-484, 2013.

20. Yeh $\mathrm{CH}$, Yang YY, Huang YF, Chow KC and Chen MF: Induction of apoptosis in human Hep3B hepatoma cells by norcantharidin through a p53 independent pathway via TRAIL/DR5 signal transduction. Chin J Integr Med 18: 676-682, 2012

21. Agnihotri N, Kumar S and Mehta K: Tissue transglutaminase as a central mediator in inflammation-induced progression of breast cancer. Breast Cancer Res 15: 202, 2013.

22. Yakubov B, Chelladurai B, Schmitt J, Emerson R, Turchi JJ and Matei D: Extracellular tissue transglutaminase activates noncanonical NF- $\kappa \mathrm{B}$ signaling and promotes metastasis in ovarian cancer. Neoplasia 15: 609-619, 2013.

23. Caffarel MM, Chattopadhyay A, Araujo AM, Bauer J, Scarpini CG and Coleman N: Tissue transglutaminase mediates the pro-malignant effects of oncostatin $M$ receptor overexpression in cervical squamous cell carcinoma. J Pathol 231: 168-179, 2013.

24. Wang Z, Perez M, Caja S, Melino G, Johnson TS, Lindfors K and Griffin M: A novel extracellular role for tissue transglutaminase in matrix-bound VEGF-mediated angiogenesis. Cell Death Dis 4: e808, 2013. 